\title{
Change in heart period: A function of sensorimotor event timing within the cardiac cycle
}

\author{
BEATRICE C. LACEY and JOHN I. LACEY \\ Fels Research Institute, Wright State University School of Medicine, Yellow Springs, Ohio 45387
}

\begin{abstract}
In a fixed-foreperiod reaction time experiment with 66 male college students, the heart rate for the same cardiac cycle within which the imperative stimulus occurred was slowed. The slowing was greater for imperative stimuli presented early in the cardiac cycle than late. The "monotonic" (linear) trend was highly significant, $\mathrm{p}<10^{-8}$. There was also a significant "bitonic" (quadratic) trend, $p<.01$. Three subgroups of subjects, split on the basis of heart rate level, each showed significant trends with significant differences in the trend-components among groups. Analyses of ready signal presentations and of a control point in the intertrial interval revealed no comparable effects. The results were replicated in a second experiment in which 20 male college students self-initiated tachistoscopic exposures. In this experiment, the next cardiac cycle, subsequent to the cardiac cycle in which the self-initiated response occurred, was also shown to exhibit systematic modification with changes in the temporal placement of the response within the preceding cycle. The phenomena described are strikingly parallel with results obtained in animals upon direct stimulation of the vagus and carotid sinus nerves, and in man upon stimulation of the carotid sinus with neck suction. The results constitute the first demonstration in intact man of differential modification of heart period with temporal variations of the placement of "significant" sensorimotor events within the cardiac cycle.
\end{abstract}

Studies of what have come to be called "cardiac cycle effects" usually have dealt with the dependence of sensorimotor activity, typically reaction time or signal detection, either on phasic variations of heart rate or on the time of occurrence within the cardiac cycle at which a stimulus or response occurs. This report, however, describes a new "cardiac cycle effect" in which the duration of a single cardiac cycle itself is significantly related to where in that cycle, or in the just-preceding cycle, certain brief sensorimotor events occur.

Our first observation of the effect was fortuitously made in an analysis of a fixed-foreperiod reaction time experiment, undertaken to study the relationship of anticipatory cardiac decelerations to speed of response. Typically, in such experiments, an anticipatory heart rate deceleration occurs during the foreperiod, reaching a nadir when the stimulus is presented (e.g., J. I. Lacey \& B. C. Lacey, 1970). In such experiments, consistent, although rather low, correlations have been found between cardiac deceleration and reaction time, with reaction time being faster the greater the deceleration. We observed that the heart rate, for the very cardiac cycle

This research was supported by a grant ( $\mathrm{MH}$ 623) from the National Institute of Mental Health. A preliminary report of part of this paper was made at the 1972 annual meeting of the Society for Psychophysiological Research (see B. C. Lacey and J. I. Lacey, 1973). in which the stimulus occurred, depended in part on where in the cardiac cycle the stimulus happened to fall. Stimuli which occurred early in the cycle were associated with slower heart rates than those which fell late in the cycle. The analyses described in Experiment 1 , a fixed-foreperiod reaction time experiment, were undertaken to study this phenomenon in detail.

Experiment 2 extended and generalized the results of Experiment 1 in a very different context. This second experiment was concerned with the relationship of cardiac cycle duration to the timing of selfinitiated tachistoscopic exposures in a pilot study for a signal detection experiment.

\section{EXPERIMENT 1}

\section{Method}

Subjects. Seventy-nine male Antioch College freshmen served as paid volunteers. The data for 66 of the subjects were used in this study, 13 subjects having been excluded prior to any analysis of their data. Reasons for exclusion were: three subjects fell asleep; four had excessive data loss due to movement; two failed to follow instructions; one coughed excessively; one had a cardiac abnormality; recording difficulties resulted in data loss for two additional subjects.

Apparatus. An electrocardiogram (EKG), heart rate (HR), respiration, and digital systolic blood pressure were recorded on a Grass Model 7 polygraph. A Fels cardiotachometer, a Fels pneumograph, and a Biophysical Electronics blood pressure monitor (Model Fels) were used to measure these variables. 
Beat-by-beat heart period (HP) in milliseconds was also recorded on punched paper tape by SETAR, an elapsed time recorder (Welford, 1966). Through suitable circuitry, SETAR was triggered by each R-wave of the electrocardiogram. The time of occurrence of the ready and imperative stimuli and of the subject's motor responses to these stimuli (see below) were also recorded on the paper tape, to the nearest millisecond, and on the polygraph.

Stimulus presentation was controlled by digital logic equipment in conjunction with a photoelectric tape reader. Ready and imperative stimuli were displayed to the research participant on an Industrial Electronic Engineers one-plane, in-line, display positioned at eye level $6 \mathrm{ft}$ in front of the subject.

Procedure. The experiment was conducted in the winter months, November to February, during afternoon hours only. The experimental room was sound deadened and was maintained at $24^{\circ} \pm .5^{\circ} \mathrm{C}$ and $42 \% \pm 4 \%$ relative humidity. A free-field 60 -dB masking noise produced by a Grason-Stadler noise generator was used throughout the session.

The subjects were seated in a Barcalounger and were told that heart rate, respiration, and blood pressure activity would be recorded during a preliminary rest period and while they participated in a reaction time experiment. They were cautioned that the recording instruments were sensitive and that they were to refrain from unnecessary movements. A telegraph key, supported by a flat board mounted on the right arm of the Barcalounger, was positioned so that the subject could comfortably rest the index and middle fingers of his right hand on the key. EKG plate electrodes were attached using Lead II. The respiratory transducer, a mercury-filled Whitney strain gauge, was positioned at the base of the sternum. Two Infratron pickups, one for measuring blood pressure and one for monitoring the unoccluded pulse, were attached to the terminal phalanges of the second and fourth fingers of the left hand. An occluding cuff was positioned at the base of the second finger. Only the cardiac data, however, are considered in this report.

The experiment began with a 10-min period during which the subject rested with eyes closed. Overhead illumination was dimmed slightly and the subject was asked to remain quiet and relaxed. The subject was aware that he could be seen and heard from the instrument room.

At the end of the rest period, instructions were given for the reaction time experiment. The subject was told that each trial would be initiated by the appearance of a ready signal-a green circle in the display box in front of him. He was then to: (1) press down on a telegraph key with the index and middle fingers of his right hand, (2) hold the key down until the imperative signal, a white cross, was superimposed on the green circle, and (3) respond as quickly as possible to the white cross, by releasing the key. The subject was informed that if the key was released prematurely the light would go out and the trial would have to be repeated. He also was told that there would be catch trials in which the imperative signal would not appear. In that case, he was not to release the key until the ready signal was turned off. The subjects were then given nine practice trials before the experiment began. Reaction times were not reported to the subject, but he was aware that the times were being recorded.

Design. A fixed foreperiod of $4 \mathrm{sec}$ was used, timed from key depression following the ready signal to the onset of the imperative signal. There were 133 trials, 108 of which were included in the analysis. Excluded were 12 interspersed catch trials, 12 trials which followed catch trials, and the first trial in the experiment. Intertrial times, defined as time from offset of the imperative stimulus to onset of the next ready signal, were in a prearranged haphazard order. They varied, in integer units, from 6 to $11 \mathrm{sec}$, each represented equally often. The order of intertrial times for the first half of the trials was reversed for the second half. During catch trials, the ready signal remained on for $5 \mathrm{sec}$. For all other trials, the ready and imperative signals were terminated by the subject's response to the imperative stimulus.

Data quantification. The digital paper tapes produced by
SETTAR were computer analyzed to determine for each subject on each trial: (1) the absolute time of stimulus occurrence, that is, the time in milliseconds from $R$-wave to imperative stimulus, (2) the R-R interval in milliseconds for that cardiac cycle, and (3) the relative time of stimulus occurrence, that is, the ratio of (1) to (2). For control or comparison purposes, identical analyses were performed using two other target times: time of occurrence of the ready signal and a control time during the intertrial interval, precisely $4 \mathrm{sec}$ prior to the ready signal. All polygraph records were inspected for artifacts in the cardiac recordings, and any trials in which artifacts occurred were eliminated from the computer analyses via control cards.

For each subject, and separately for each of the three "events" (the imperative stimulus, the ready signal, and the control point), two functions, relating HP to time of event occurrence, were formed from the HPs for the cardiac cycle in which the event occurred. One function was based on the relative time of event occurrence, as previously defined. The median HPs were determined for each of 10 class intervals of .1 (i.e., median HP as a function of 10ths of the duration of the cardiac cycle). The second function was based on the absolute time of event occurrence, as previously defined, using a class interval of $100 \mathrm{msec}$. Such individual curves will be called "median HP curves." Group curves were then formed, for illustrative purposes only, by averaging the median HP curves for the 66 subjects. Medians were used for the individual curves to secure representative measures of central tendencies that would not be influenced by marked skews in within-individual distributions. In accordance with this usage, nonparametric tests of significance are used in the analysis of results.

\section{Results}

Heart period and relative time of stimulation. Figure 1 shows the group curves of HP as a function of the decile of the cardiac cycle in which each of the three events occurred. The difference in height of the three curves shown in this figure reflects the changes in $H R$ seen in reaction time experiments. Heart rate decelerated (HP increased) from a control point in the intertrial interval in anticipation of the ready signal. A larger preparatory deceleration then occurred between the ready signal and the imperative stimulus. As can be seen, however, the magnitude of this deceleration was dependent on where in the cycle the stimulus happened to occur. Stimuli occurring early in the cycle resulted in greater prolongation of that cycle than stimuli occurring late in the cycle. For example, for stimuli falling in the first decile, the average change in HP from the ready to the imperative stimulus was approximately $38 \mathrm{msec}$ (from 826 to $864 \mathrm{msec}$, a change of 3.2 beats $/ \mathrm{min}$ ), whereas, for stimuli falling in the 10 th decile, the average change was approximately $20 \mathrm{msec}$ (from 826 to $846 \mathrm{msec}$, a change of 1.7 beats/min). Figure 1 suggests some nonlinearity, since slightly longer HPs were associated with stimuli falling in the 3rd and 4th deciles than in the 1st and 2 nd.

Ferguson's nonparametric trend test (Ferguson, 1965) was applied to each of the three sets of median HP curves underlying Figure 1. This relatively unfamiliar trend test is a straight-forward extension of 


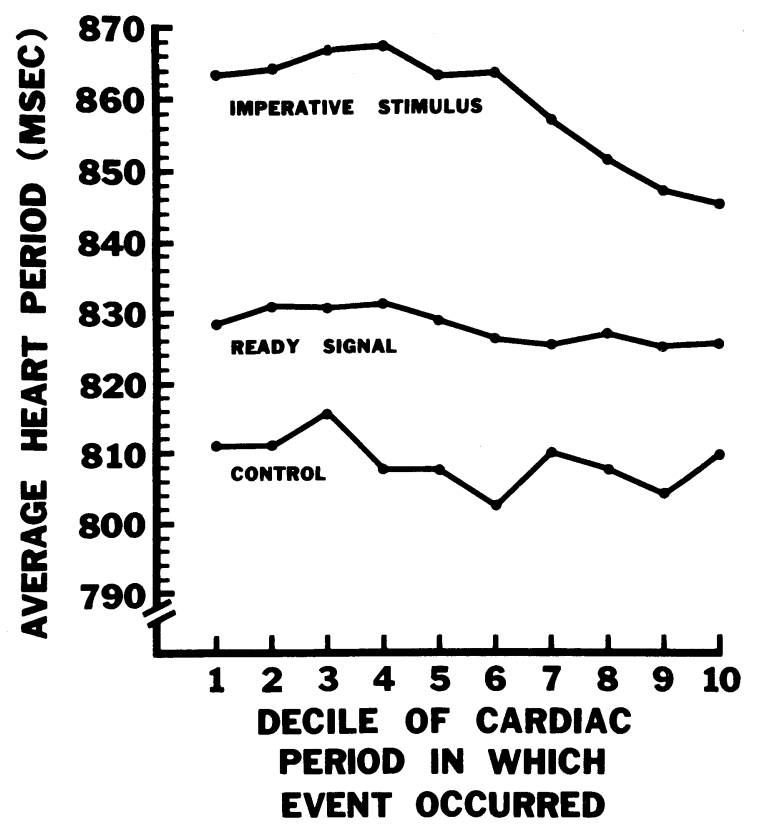

Figure 1. Relationship of concurrent heart period to relative cycle time of event occurrence. Ordinate is average of individual median heart periods. $\mathbf{N}=66$.

the statistically elegant tau (Kendall's rank order correlation). Kendall's S statistic, which underlies tau, is computed for each subject in the course of testing trends. The availability of the $\mathrm{S}$ statistic for each subject enabled later comparisons between subgroups of subjects and between events. For those not familiar with this test, it should be noted that in each case the overall significance of results was confirmed by means of the Friedman two-way analysis of variance by ranks (Siegel, 1956).

The "monotonic" (linear) trend for the imperative stimulus was highly significant, $p<10^{-8}$. The "bitonic" (quadratic) trend was significant also, $\mathrm{p}<.01$. Significant trends were not found, however, either for the ready signal or the control point. Thus HP was independent of the time of presentation of the ready signal and of the time within the cardiac cycle at which the control point fell, but it varied systematically with time of presentation of the imperative stimulus.

Direct comparisons of the three events showed that the monotonic trend for the imperative stimulus differed significantly from that for both the ready signal and the control point curves $\left(\mathrm{p}<10^{-5}\right.$ in each case). The imperative stimulus differed significantly in bitonic trend as well, when compared either with the ready signal curve $(p<.01)$ or with the control point curve $(p<.05)$. Ready signal and control point trends did not differ from each other. Differences in each case were tested, by Wilcoxon's (Wilcoxon \& Wilcox, 1964) two-tailed paired replicates test, from paired comparisons of the statistic S.
Effect of heart rate level. Figure 2 shows the group averages of the median HP curves for the imperative stimulus for three subgroups of subjects. The subgroups were secured by arranging the subjects in the order determined by the individual's median HR for the cardiac cycles in which imperative stimuli occurred. The "slow HR" subjects had median HPs between 907 and 1,198 msec, the "middle HR" subjects, between 801 and $902 \mathrm{msec}$, and the "fast HR" subjects, between 660 and 800 msec. Monotonic trends were significant for each group, each group showing a tendency for longer HPs with early than with late stimulation; $\mathrm{p}<10^{-8}, \mathrm{p}<10^{-5}$, and $\mathrm{p}<.01$, for fast, middle, and slow HR groups, respectively. There was also a significant bitonic trend for slow HR subjects, $\mathrm{p}<.001$.

Comparable analyses were made for both the ready signal and for the control point, using the same subject grouping as in Figure 2 . In all but one instance there were no significant trends for either ready or control data. The single exception to this was for the middle HR group's ready signal curve, for which the monotonic trend was just significant, $\mathrm{p}<.05$.

While the composition of the groups changed very little when the classification of the subject as "slow," "middle," or "fast" HR was based on median HR at the ready signal, or at the control point, there was

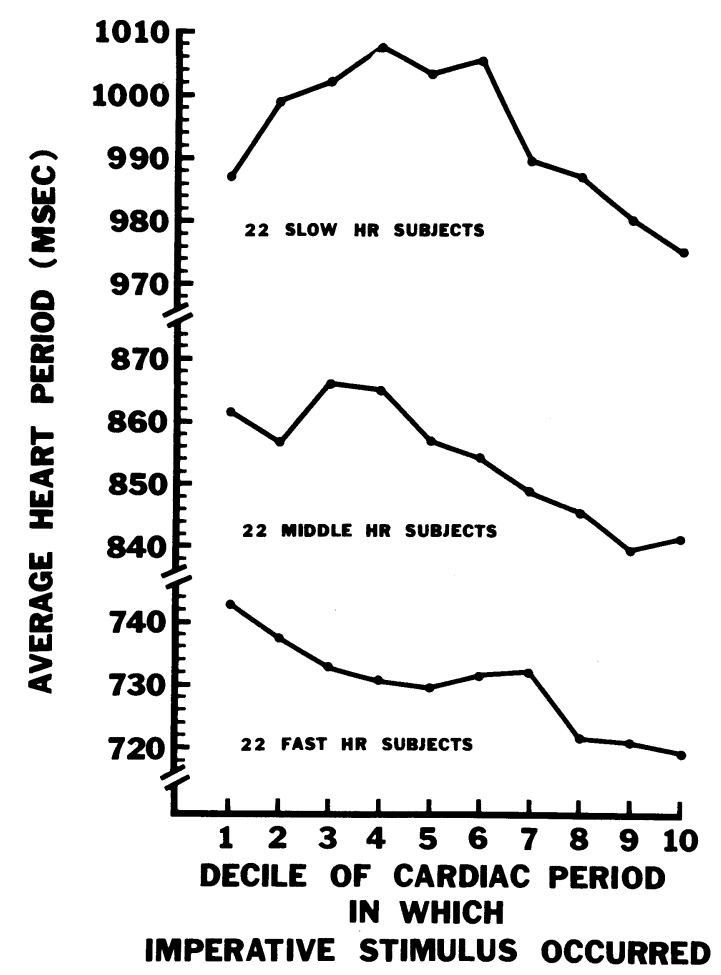

Figure 2. Relationship of concurrent heart period to relative cycle time of imperative stimulus for each of three groups of subjects separated by heart rate level. 
some cross-over between adjacent groups. Consequently, analyses for the imperative stimulus were repeated with group splits based on ready signal HR and with group splits based on control-point HR. Ready signal analyses were also repeated with group splits based on ready signal HR, and control point analyses were repeated with control point splits. The results were virtually unchanged. All previously significant trends associated with presentation of the imperative stimulus remained significant, while no trends for any group were significant in either ready signal or control-point analyses.

While each HR group showed significant changes in $\mathrm{HP}$ as a function of relative time of stimulus presentation within the cardiac cycle, the functions appear to differ somewhat among groups. Fast HR subjects show an almost monotonic decreasing trend from the first decile, middle HR subjects from the third, and slow HR subjects from the fourth or sixth decile. Thus, the slower the HR group, the later the appearance of a regularly decreasing function and the more likely it is that an earlier increasing function will appear. The significance of the differences among the three HR groups in both monotonic and bitonic trend was computed from individual $\mathrm{S}$ statistics by means of the Kruskal-Wallis one-way analysis of variance test (Siegel, 1956). There was a significant difference $(p<.05)$ for monotonic trend, but only when the HR split was based on median $\mathrm{HR}$ at the imperative stimulus. It was not significant when the split was based on either control or ready signal median HRs.

It seemed likely, however, that the difference among HR groups was only marginally significant because of the similarity in group trends for stimuli presented in the last half of the cardiac cycle (deciles 6 through 10). The big difference among groups seemed to be confined to the first half of the cycle. Separate trend analyses were done, therefore, for the first and second five deciles of the cardiac cycle.

Only the fast HR group showed a significant monotonic trend $(p<.01)$ during the first half of the cycle. The middle HR group showed a significant $(p<.05)$ "tritonic" (cubic) trend and the slow HR group showed no significant trend. Kruskal-Wallis tests showed a significant difference among groups in monotonic trend during the early part of the cycle, whether group composition was based on HR at stimulus $(p<.02), H R$ at control point $(p<.02)$, or $\mathrm{HR}$ at ready signal $(\mathrm{p}<.05)$. There were no significant differences in bitonic or tritonic trends.

During the second five deciles, each group showed a significant decreasing monotonic trend $(p<.001$ for both fast and slow HR subjects, $p<.01$ for middle HR subjects). There were no significant differences among groups in trend during the latter half of the cycle.
Real time analyses. In describing HP as a function of relative time of event occurrence, as was just done, the data from all artifact-free trials for all subjects were used. But, in analyzing HP as a function of the absolute elapsed time in multiseconds from $\mathrm{R}$-wave to event a considerable reduction in utilizable data must be imposed. This is because in real time analyses, unlike the case in relative time analyses, the probability of event occurrence is not equal for all values on the abscissa. In real time analyses, in order to assure equal probability of event occurrence, it is necessary to use intervals on the abscissa, from $\mathrm{R}$-wave to event, that are no greater than the shortest cardiac cycle found at event occurrence, over all subjects and all trials. If this is not done, the varying probability of event occurrence at different elapsed times from $\mathrm{R}$-wave will produce a biased and artifactual functional relationship.

Consider, for example, a single subject whose HPs, for the cycle in which an event occurred, vary between 800 and $999 \mathrm{msec}$ (HRs between 75 and 60 beats per minute). Elapsed times from R-wave to event could never be greater than $999 \mathrm{msec}$ for this subject. For another subject, however, whose HPs vary from 900 to $1,100 \mathrm{msec}$, many trials could show R-wave-to-event times greater than $999 \mathrm{msec}$. The combined data for these two subjects necessarily would show long HPs for long elapsed times between R-wave and event. Similarly, within a single subject, long elapsed times would necessarily be associated with longer HPs. If, however, analysis is restricted as described, then an artifactual relationship between elapsed time and HP will be avoided.

The cutoff point used in the real-time analyses was $599 \mathrm{msec}$, since it was the upper limit of the highest 100 -msec class interval which satisfied the criterion.

Despite this restriction, a modest decreasing monotonic trend, $\mathrm{p}<.01$, was found during the first six 100-msec class intervals for the imperative stimulus (see Figure 3). Again, comparable analyses for the ready signal and the control point revealed no significant trends.

Real-time analyses by HR groups are shown in Figure 4, and are based on the same classification used in Figure 2. No significant trends were found in either the middle or the slow HR groups, but the monotonic trend for fast $\mathrm{HR}$ subjects was significant, $\mathrm{p}<10^{-4}$. The group differences are not surprising in view of the fact that the first six intervals include a much larger portion of the total cardiac cycle for fast HR subjects than for the other groups. Furthermore, analyses of the first five deciles had shown no significant monotonic trends for the slower groups during the first half of the cycle.

By testing directly the significance of the difference in trends among groups, frequency dependency 


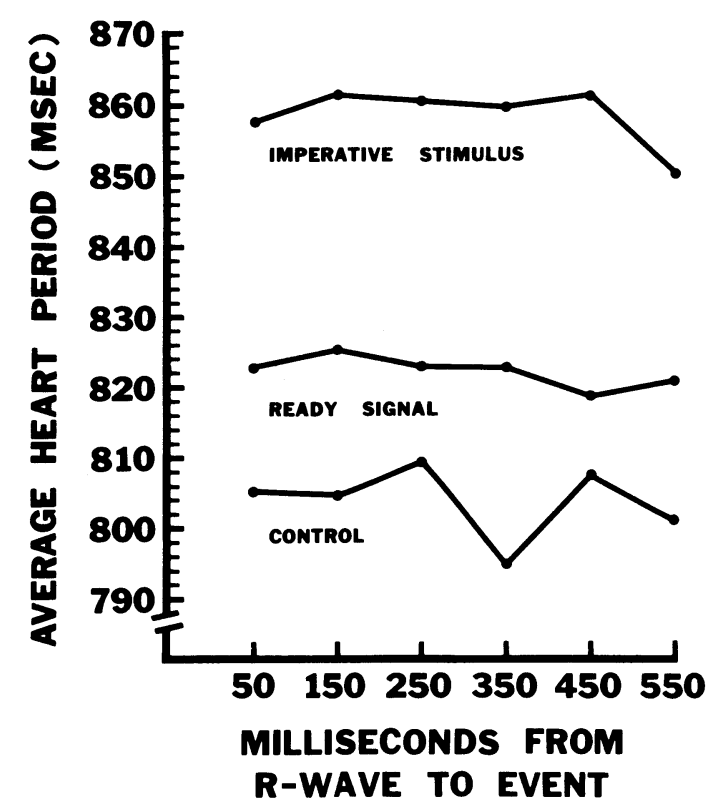

Figure 3. Relationship of concurrent heart period to absolute cycle time of event occurrence. $N=66$. Midpoints of 100 -msec class intervals are noted on the abscissa.

of the basic effect was again shown. There was a significant difference in monotonic trend $(p<.01)$, by the Kruskal-Wallis one-way analysis of variance test, using the individually obtained $\mathrm{S}$ statistics as

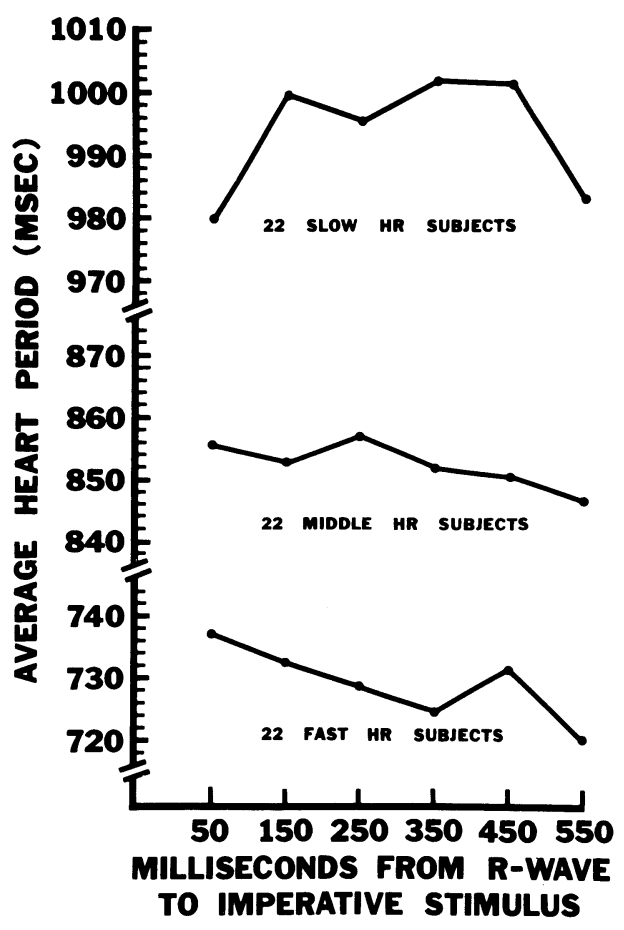

Figure 4. Relationship of concurrent heart period to absolute cycle time of imperative stimulus for each of three groups of subjects separated by heart rate level. Mid-points of $100 \mathrm{msec}$ class intervals are noted on the abscissa. entries, among slow, middle, and fast HR groups for the first six 100-msec class intervals. This result also is compatible with the relative-time analyses which showed that differences among groups were significant in the early part of the cycle rather than in the later part.

\section{Discussion}

While we have not yet performed experiments to elucidate the neural and biochemical mechanisms underlying the demonstrated dependence of cardiac cycle duration on the time of event occurrence within the cardiac cycle, the evidence from over four decades of experiments in cardiovascular physiology (see, e.g., Levy, 1977, for a review and citation of the relevant literature) clearly implicates the vagus as the final common pathway. Vagal control of the heart is prepotent over sympathetic control, and vagal, but not sympathetic, control can produce such prompt changes in HP as we have reported. Moreover, the effects we have reported are strikingly congruent with the now well-documented fact that there are temporal variations in the susceptibility of the cardiac pacemaker to vagal stimuli. Such timedependency is not detectable with variations in sympathetic control of the heart.

Temporal dependency was reported in a classic paper in 1934 by Brown and Eccles and is the subject of much recent work by Levy and his colleages (e.g., Levy, Iano, \& Zieske, 1972; Levy, Martin, Iano, \& Zeiske, 1969, 1970) and by others (e.g., Dong \& Reitz, 1970; Reid, 1969). In acute animal preparations, these investigators have shown that electrical stimulation of the vagus early in the cardiac cycle prolonged that cycle but had little effect on the subsequent cycle. Stimulation later in the cycle, about the end of the T-wave, failed to affect that cycle but prolonged the next one, the more so the later the stimulation. The mechanism underlying this temporal dependency will be discussed later in this paper.

The parallelism between these effects on the concurrent cycle of the timing of direct electrical stimulation of the vagus and those which we found with the presentation of the imperative stimulus was provocative. We therefore wished to see if our results could be generalized and extended. Of particular concern was whether the parallel to the animal experiments would continue to emerge when the cardiac cycle subsequent to the cycle in which an event occurred was studied.

\section{EXPERIMENT 2}

\section{Method}

Subjects. Twenty-two male Antioch College students, all but three of them freshmen, served as paid volunteers. The data for 20 of the subjects were used, those of 2 having been discarded because of equipment failure. 
Apparatus. An electrocardiogram, heart rate, and respiration were measured and recorded as described in Experiment 1. Skin potential was also recorded for purposes other than those covered in this report. The subject activated a display positioned at eye level, $6 \mathrm{ft}$ in front of him, by lifting a finger from a touch plate. The display consisted of a clockface in which clear red Monsanto MV5022 light emitting diodes (LED), slightly recessed in 1/4-in.diam apertures, were arranged in labeled clock positions, 1 through 12, around the perimeter of a circular area 2 in. in diameter. An additional LED was positioned in the middle of the circle. The display was contained in a black box, the face of which was 10-7/8-in. in height and 7-7/8 in. in width. The box also contained a rectangular area, above the clock face, in which numerals could be shown by an Industrial Electronic Engineers one-plane, in-line display. The LEDs were matched for brightness and were maintained at $750 \mathrm{fL}$ at $20 \mathrm{~mA}$. Rise and fall times were $1 \mathrm{nsec}$, which resulted in instantaneous display illumination upon subject response.

Procedure. The experiment was conducted during afternoon hours of May and June. The experimental room, seating arrangement, masking noise, electrode attachment, and cautions against excessive movement were, for the most part, as in Experiment 1. However, laboratory temperature was maintained at $22^{\circ} \pm .5^{\circ} \mathrm{C}$, relative humidity at $41 \% \pm 5 \%$, blood pressure was not recorded, and electrodes for measuring skin potential were attached, one on the left thenar eminence and one on an inactive ulnar site on the left arm. Overhead illumination was maintained at $10 \mathrm{fc}$, as measured at the rear shelf of the Barcalounger. A touch plate supported by the board on the right arm of the Barcalounger was placed in a position which enabled the subject to comfortably rest one finger on it.

Each subject was told that some physiological aspects of difficult visual perception were being studied, that we were primarily interested in his ability to see things quickly. He also was instructed that: (1) lights on the clockface would be flashed on for very brief periods of time and his job was to report which light was off; (2) the correct answer would be displayed in the rectangle above the clockface several seconds later (actual time was $2 \mathrm{sec}$; both time computation and display were controlled by digital logic equipment); (3) he would self-initiate each trial when ready to do so by gently lifting his finger from the touch plate on which it rested, having first fixated the center light and inhibited blinking; (4) trials would be grouped in blocks of 25 each, with a short rest between blocks. Exposure times were announced at the beginning of each block, and were kept constant within blocks. From block to block, exposure times were changed, in a futile attempt to secure about $50 \%$ correct responses in a block.

The procedure was first demonstrated with trials initiated by the experimenter, beginning with a $900-$ msec exposure time. Six additional trials at each of several exposures followed. Exposure times ranged from 850 to $600 \mathrm{msec}$, the time being decreased successively by 50 -msec steps. This was followed by a practice session in which the subject initiated each trial. Exposure times started at $600 \mathrm{msec}$ and dropped by $50 \mathrm{msec}$ for each trial. The subject was informed of each exposure time. A given time was repeated if the subject made an incorrect response, and the procedure continued until there were two consecutive failures. The first block of 25 trials was used as a practice block, during which exposure times started with the shortest time that had previously yielded a correct response. The time was increased or decreased during the block in an attempt to maintain between $50 \%$ and $60 \%$ correct responses. Following the practice block, exposure time was altered between blocks only. There was a total of seven experimental blocks of 25 trials each, a total of 175 trials in all.

Data quantification. SETAR tapes were computer analyzed to determine for each subject on each trial: (1) time in milliseconds from $R$-wave to subject response, (2) R-R interval for the response cycle, (3) relative time of subject response, as defined as Experiment 1 and (4) R-R interval for the subsequent cycle. Trials con- taining artifacts were eliminated, as in Experiment 1. Median HP curves and average group curves were derived, as in Experiment 1 , for both the response cycle and the subsequent cycle, grouped both by quintile of response occurrence and by 100 -msec class intervals. Quintiles, rather than deciles, were used in these analyses as they produced slightly smoother group curves.

\section{Results}

Heart period of the concurrent cycle and relative time of stimulation. The results obtained in this experiment were very similar to those of Experiment 1. Figure 5 shows that the length of the cycle in which the self-initiated response occurred varied as a function of cycle time of response. The curve was obtained by averaging the median HP curves of the 20 subjects. The dashed line indicates the group average of median HP for the cycle just preceding the one in which the response was made.

In interpreting this and subsequent figures, it should be noted (paper in preparation) that systematic cardiac changes occurred in this experiment like those found in reaction time experiments. A highly significant beat-by-beat deceleration was found preceding the execution of the response. It is not known exactly how early the deceleration may have begun since analyses were limited to the six cardiac cycles before the subject made his overt response. However, the analysis period was sufficiently long to

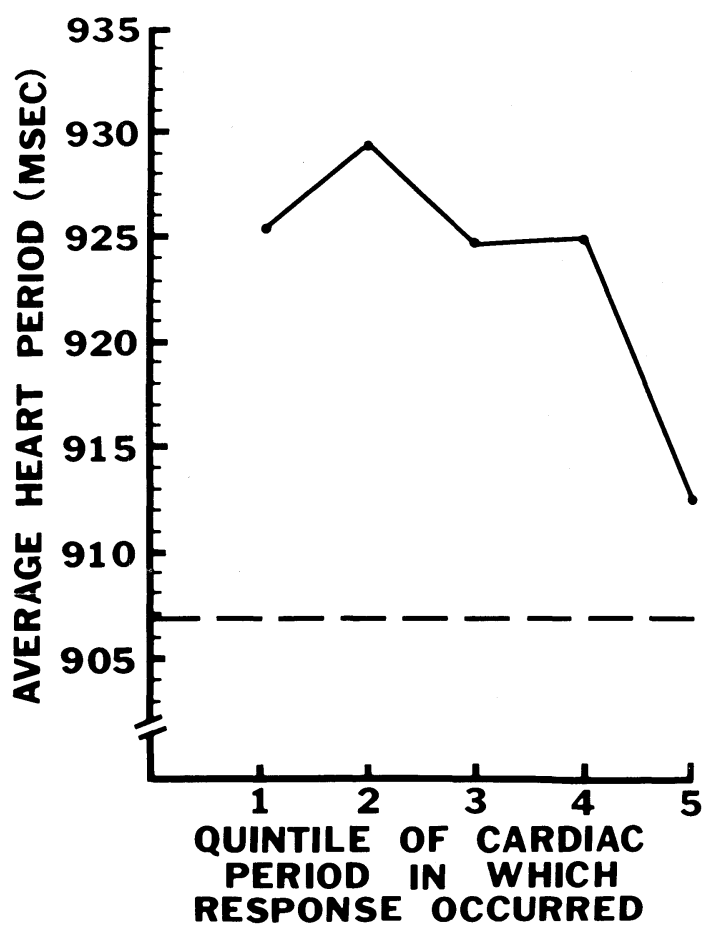

Figure 5. Relationship of concurrent heart period to relative cycle time of subject response. Dashed line indicates group average of median heart periods for the single preceding cardiac cycle. $\mathbf{N}=\mathbf{2 0}$. 


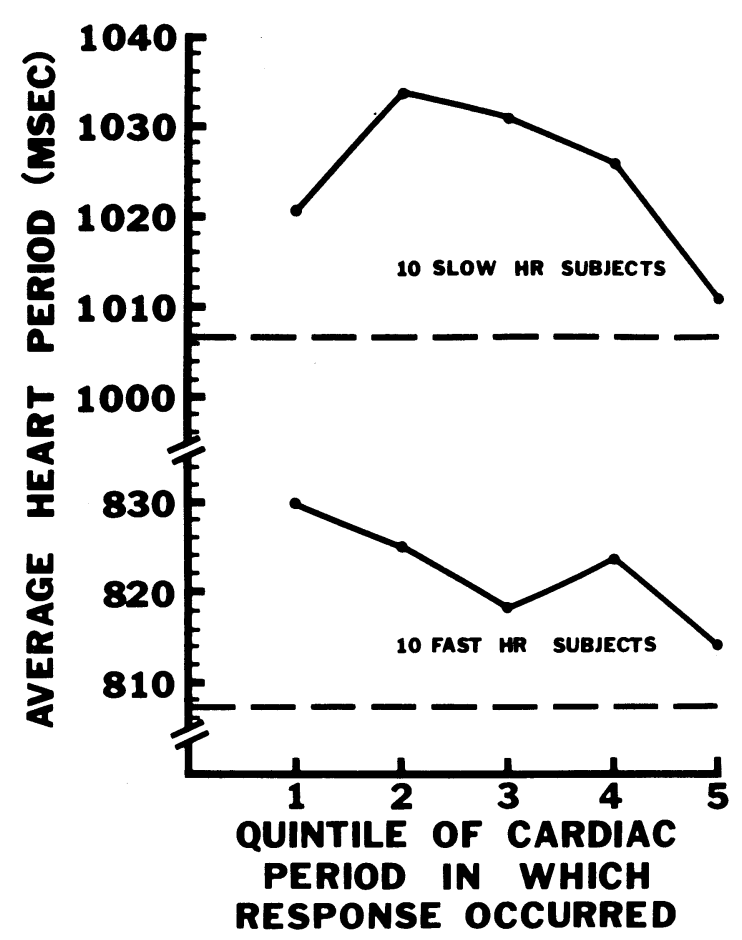

Figure 6. Relationship of concurrent heart period to relative cycle time of subject response for subjects grouped by heart rate level. Dashed lines indicate average of median heart periods for the single preceding cardiac cycle for each group.

show that decleration began as much as $8 \mathrm{sec}$ (in slow HR subjects) before the overt response. It reached a nadir with the self-initiated tachistoscopic exposure, and was followed by a rebound acceleration in which HRs exceeded the level before the deceleration began.

The difference in height, then, of the two curves in Figure 5 reflects the end phase of a period of beatby-beat deceleration which commences well in advance of a subject's intention to make a response. There was a much greater increase in HP from the just preceding cardiac cycle when the response occurred early in the cycle than when it occurred late. The monotonic trend was significant, $\mathrm{p}<.01$.

While there is some indication of the same bitonicity seen in Experiment 1, this trend was not significant for the group as a whole. As in Experiment 1 , however, a bitonic trend was pronounced for slower HR subjects, as shown in Figure 6, which depicts the concurrent cycle effects for subjects split into slow and fast HR groups of 10 subjects each. The 10 slow HR subjects had median HPs from 930 to $1,136 \mathrm{msec}$ (for the cycle preceding the overt response). The 10 fast subjects had median HPs from 694 to $910 \mathrm{msec}$. The dashed lines below each group curve depict that group's average median HP for the preceding cycle. Trends were similar to those obtained in the first experiment. Fast HR subjects again began the monotonically decreasing portion of the curve earlier in the cycle than did slow HR subjects. The monotonic trend was significant for fast HR subjects, $p<.01$. The monotonic trend was not significant for slow HR subjects, but the bitonic trend was, $\mathrm{p}<.01$. Wilcoxon's two-tailed unpaired replicates test revealed significant differences in bitonic trend between the two groups $(p<.05)$, but there were no significant differences in monotonic trend.

Heart period of the subsequent cycle and relative time of stimulation. Figure 7 shows the effect of time of response on the subsequent HP. The dashed line once again indicates HP for the single cardiac cycle prior to response. The HP of the cycle following response is markedly influenced by where in the previous cycle the event occurred. When it occurred early in the previous cycle, recovery toward faster HR levels was well under way by this next cycle, the more so, the earlier the response time. When response occurred late in a cycle, the HR of the subsequent cycle failed to even reach the preresponse level; that is, the process of anticipatory deceleration was continued for at least one more cardiac cycle. The monotonic trend was significant, $\mathrm{p}<10^{-5}$.

Separate curves for slow and for fast HR subjects are shown in Figure 8. Both curves show significant monotonic trends, $\mathrm{p}<10^{-4}$ for slow HR subjects and $\mathrm{p}<.01$ for fast HR subjects. The trends for the two groups do not differ significantly. Slow HR subjects,

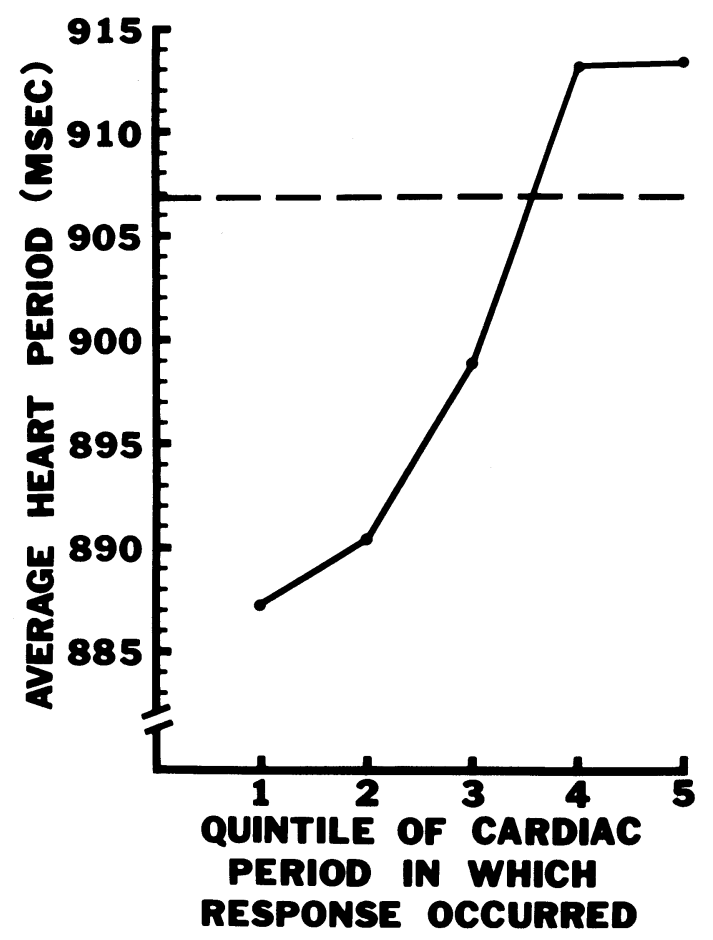

Figure 7. Relationship of subsequent heart period to relative cycle time of subject response. Dashed line indicates group average of median heart periods for the single cardiac cycle preceding the response. $\mathrm{N}=\mathbf{2 0}$. 


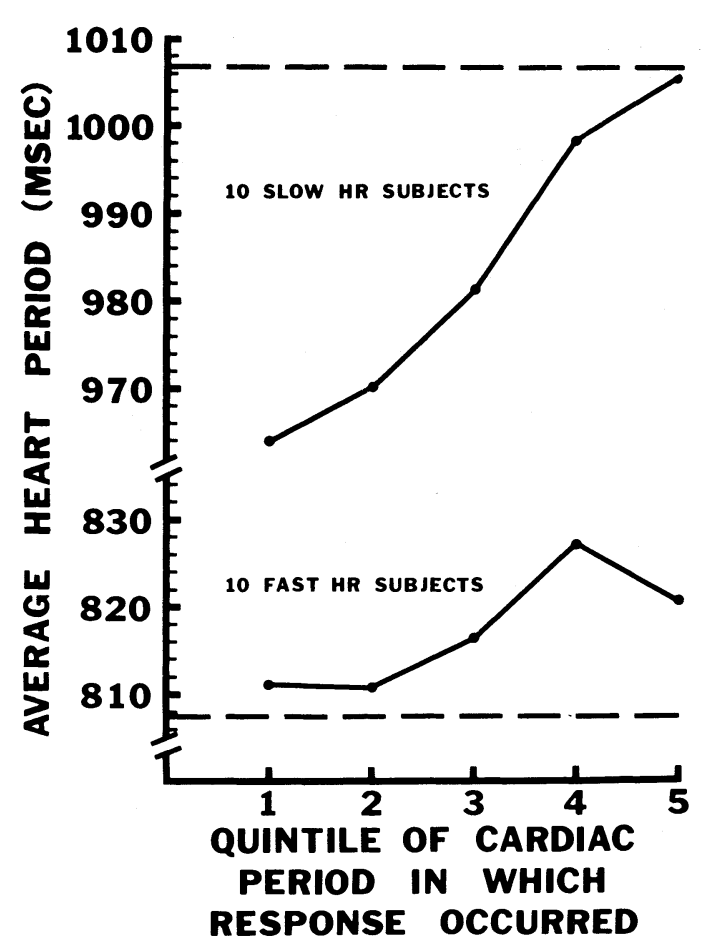

Figure 8. Relationship of subsequent heart period to relative cycle time of subject response for subjects grouped by heart rate level. Dashed lines indicate average of median heart periods for the single cardiac cycle preceding the response for each group.

however, show a much greater differential effect of response time. Their HRs have already begun to recover and are faster than those of the preresponse cycle, the more so, the earlier the event occurred. For the fast HR subjects, HPs are still relatively long as compared with the preresponse cycle. Comparisons based on the median HP of the subsequent cycle (irrespective of cycle time of response), indicate that, by this cycle, slow HR subjects show a greater absolute and percentage recovery toward preresponse HP than do fast HR subjects, $p<.02$ by the Wilcoxon rank sum test (Wilcoxon \& Wilcox, 1964).

Real-time analyses. Analyses of the effect of absolute time of event occurrence on both the concurrent and subsequent cycles were even more restricted in this experiment than in Experiment 1. Two subjects had some sufficiently fast HRs (minimum HPs of 536 and $539 \mathrm{msec}$, respectively) that we were limited to a group analysis of the first five $100-\mathrm{msec}$ class intervals only. The results were again comparable to those obtained in the relative time analyses. Significance, however, was restricted, in analyses of the concurrent cycle, to the monotonic trend for fast HR subjects only, and in analyses of the subsequent cycle, to the monotonic trend for the total group, $\mathrm{p}<.05$ in both instances.

\section{Discussion}

The effects of time of event occurrence on the HP of the concurrent cycle, which were demonstrated in Experiment 1, were generalized to the very different conditions of the second experiment. It was again found that the timing of a punctate event within a single cardiac cycle can differentially affect the duration of that same cycle. Heart periods are slower when events occur early in the cycle, rather than late, and slow and fast HR subjects differ somewhat in this effect. In addition, we were able to strengthen the analogy to results obtained in acute animal experiments by the demonstration that responses occurring late in the cardiac cycle were associated with a slower subsequent HR than were early responses.

\section{GENERAL DISCUSSION}

The results of both experiments provide, to the best of our knowledge, the first demonstration in intact $\operatorname{man}^{1}$ that punctate sensorimotor events produce phenomena analogous to those produced by: (a) direct electrical stimulation of the vagi in cats and dogs (see already cited references); (b) electrical stimulation of the carotid sinus nerves in dogs (Levy \& Zieske, 1972); and (c) stimulation in man of baroreceptors by neck suction (Eckberg, 1976; Eckberg, Abboud, \& Mark, 1976). Variations in the timing, within the cardiac cycle, of punctate sensorimotor events in human experiments, of direct vagal or carotid sinus nerve stimulation in acute animal preparations, and of carotid baroreceptor stimulation in man all result in systematic variations of the duration of the single cardiac cycle in which the event occurs and of the duration of the subsequent cycle. Events occurring early in a cycle prolong that cycle but have little effect on the subsequent cycle. Events occurring late in a cycle prolong the subsequent cycle, but have little effect on the concurrent cycle.

To completely reconcile the results of the two experiments reported in this paper with the basic cardiovascular data will require more detailed knowledge than is presently available of visceral efferent projections from the neocortex (and from lower neural levels). Such neocortical projections are well known (cf. Monnier, 1968, for a review), but little or nothing is known about the conditions under which these pathways become influential, or about the temporal delays imposed in the passage from neocortex to the heart. We turn, then, primarily to a consideration of the peripheral and immediately proximal mechanisms that presumably underlie our results.

The temporal dependency effects in animals have 
been attributed to the action of acetylcholine (ACh) released at parasympathetic terminals within the sinoatrial node subsequent to vagal stimulation. The $\mathrm{ACh}$ "affects the frequency of the pacemaker cell discharge principally by diminishing the slope of diastolic depolarization and by increasing the magnitude of the membrane potential during diastole" (Levy et al., 1970, p. 1259). If the slope of diastolic depolarization is diminished by the arrival of a quantity of $\mathrm{ACh}$ early in diastole, the cycle will be more prolonged than if the same quantity arrives later and thus alters the slope later in diastole. $\mathrm{ACh}$ arriving before diastole will be partially hydrolyzed before depolarization begins. The strict temporal dependency depends in part on the fact that $\mathrm{ACh}$ is rapidly hydrolyzed by the high concentrations of acetylcholinesterase in the sino-atrial node. The more sluggish removal of norepinephrine at sympathetic terminals accounts for the lack of temporal dependency for sympathetically mediated responses (Levy, 1977).

There is a delay subsequent to vagal stimulation before the effects of ACh on the pacemaker become apparent. This "latent period" is operationally defined by the time interval, prior to the end of a cycle, in which stimulation does not result in lengthening of that cycle (Brown \& Eccles, 1934; Dong \& Reitz, 1970). This interval is quite long. Dong and Reitz found it to be approximately $200 \mathrm{msec}$ in dogs. The latent period is thought to be occupied primarily in the liberation of $\mathrm{ACh}$ and its diffusion to, and effect upon, the pacemaker.

These several facts concerning the timing of the availability and effectiveness of $\mathrm{ACh}$ at the pacemaker may serve as a basis for a most tentative explanation of the differences in the effects seen in slow and in fast HR subjects. It should be noted, however, that such differences have not been described in the cited animal experiments, and Eckberg (1976) has found, in man, that differences in amplitude, but not in timing, of peak response are related to HR level. ${ }^{2}$

We assume that in humans, as in cats (Brown \& Eccles, 1934), the latent period is not dependent upon heart rate level. Let us arbitrarily suppose that the "latent period" in humans, timed from onset of the imperative stimulus, is approximately $300 \mathrm{msec}$. Then stimuli which occur $100 \mathrm{msec}$ after an R-wave could result in altered diastolic depolarization beginning $400 \mathrm{msec}$ after the $\mathrm{R}$-wave. Thus, for persons whose median HPs were around $1,000 \mathrm{msec}$, ACh could be effective in diminishing the slope of diastolic depolarization for a total of approximately $600 \mathrm{msec}$ more. However, for a person whose median HP was around $600 \mathrm{msec}$, ACh could be effective for a period of only about $200 \mathrm{msec}$. In other words: in slower HR persons, stimuli can arrive relatively later in the cycle and there still will be time, once the latent period is over, for effective modification of that cardiac cycle; but, for faster HR subjects, stimuli must arrive relatively early in the cycle in order to be able to influence that cycle at all. Our data are compatible with this formulation, for the effectiveness of a stimulus in slowing HR in Experiment 1 was not diminished until the seventh decile in the slow HR group, and until the fourth or fifth decile in the middle HR group. In the fast HR group, diminished effectiveness of the stimulus in slowing the heart could be seen as early as the second decile.

A bitonic trend was found in slow HR subjects, but not in fast HR subjects. In slow HR subjects, very early events caused less HR slowing than events occurring slightly later in the cycle. This trend may also be related to the time of arrival of $\mathrm{ACh}$ at the pacemaker. When events occur very early in a cardiac cycle, the time from event to the onset of diastolic depolarization may rather grossly exceed the latent period in slow HR subjects. Thus there would be time for some dissipation of $\mathrm{ACh}$ prior to the onset of diastolic depolarization. In fast HR subjects, shorter times to diastolic depolarization prevent such bitonicity. For these subjects, the immediate monotonic decrease in HP with increasingly later event times, primarily may reflect the later and later arrival of ACh during the effective period of diastolic depolarization.

The same factors may be involved in the differences in recovery during the subsequent cycle between slow and fast HR subjects in Experiment 2. On the average, for slow HR subjects, a longer absolute time will have elapsed between stimulation in one cycle and the beginning of diastolic depolarization in the subsequent cycle, time in which $\mathrm{ACh}$ is being hydrolyzed. It would then follow that the relative prolongation of the subsequent cycle, when compared with the preresponse beat, would be much greater for fast than for slow HR subjects, and this is what we observed. A related observation was made by Eckberg (1976) in three subjects who had very slow base HRs. In these subjects, stimulation late in a cardiac cycle ("about $0.2 \mathrm{sec}$ before the next anticipated $\mathrm{P}$ wave') were too late to prolong that cycle and too early to affect the subsequent cycle.

The experiments clearly raise an important question concerning the operation of efferent projections from the central nervous system to the heart. Not all centrally mediated sensorimotor events are capable of influencing the heart in a time-dependent manner. This follows from the failure of the ready signal in Experiment 1 to elicit such effects. The difference cannot be attributed to the associated demand of a signal for some clearly overt motor response, since both ready and imperative signals 
required such a response. The imperative signal, however, was of considerably more importance to the task at hand. It is thus likely that the event must have some special significance for the individual in order for the cycle time of its occurrence to be of consequence. It remains to be determined what attributes an event must have in order to be "especially significant." It is apparent that the timedependency phenomena are seen when event timing is under either subject control (in Experiment 2) or experimenter control (in Experiment 1). This variable, then, does not serve to define "significance."

Neither experiment permitted a determination of whether or not sensory input or motor output are separable components of the eliciting event. While subjects in Experiment 2 self-initiated responses, instantaneous (nanosecond delay) illumination of a visual display followed. It may be possible to determine whether such input is essential for development of the effect by imposing a delay of several seconds between subject response and presentation of the stimulus.

These experiments provide dramatic evidence that variations in the timing of events within a cardiac cycle differentially modify the duration of both the concurrent and subsequent cycles, and as a consequence partially determine the magnitude of the measured decelerations and recovery from these decelerations in experiments in which such decelerations are the focus of study. Additionally, the results refute two common misconceptions. The first misconception is that HR responses are relatively slow and sluggish, developing only over an interval of several seconds. The data of both experiments show conclusively that a HR response can be evoked (or at least modified) without lag, within the very cycle in which a significant event occurs, or at the next heart cycle, depending on the cycle time of the event. The second misconception is that $H R$ responses are attributable entirely to variations in metabolic demand, produced by variations in skeletal muscle activity. Such a formulation cannot begin to encompass the data reported. The data also provide a cautionary note for the study of cardiac-behavioral relationships, in that the effects on HR of cycle time of stimulus presentation almost certainly will be confounded with the effects of other factors modifying phasic changes in HR unless specific experimental and analytic techniques are used to separate the influence of time of event.

\section{REFERENCES}

Brown, G. L., \& Eccles, J. C. The action of a single vagal volley on the rhythm of the heart beat. Journal of Physiology [London], 1934, 82, 211-240.

Dong, E., JR., \& Reitz, B. A. Effect of timing of vagal stimulation on heart rate in the dog. Circulation Research, 1970, 27, 635-646.

ECKBERG, D. L. Temporal response patterns of the human sinus node to brief carotid baroreceptor stimuli. Journal of Physiology [London[, 1976, 258, 769-782.

Eckberg, D. L., Авboud, F. M., \& MARK, A. L. Modulation of carotid baroreflex responsiveness in man: Effects of posture and propranolol. Journal of Applied Physiology, 1976, 41, 383-387.

Ferguson, G. A. Nonparametric trend analyses. Montreal: McGill University Press, 1965.

Jennings, J. R., \& Wood, C. C. Cardiac cycle time effects on performance, phasic cardiac responses, and their intercorrelation in choice reaction time. Psychophysiology, 1977, 14, 297.

LACEY, B. C., \& LACEY, J. I. Variation in the temporal placement of sensorimotor events within a cardiac cycle modifies duration of that cycle. Psychophysiology, 1973, 10, 195-196. (Abstract)

LACEY, J. I., \& LACEY, B. C. Some autonomic-central nervous system interrelationships. In P. Black (Ed.), Physiological correlates of emotion. New York: Academic Press, 1970.

LACEY, J. I., \& LACEY, B. C. Heart rate-reaction time relationship varies with proximity of cardiac cycle to event, with cardiac cycle time of stimulus, and between individuals. Psychophysiology, 1973, 10, 196. (Abstract)

LEVY, M. N. Parasympathetic control of the heart. In W. C. Randall (Ed.), Neural regulation of the heart. New York: Oxford University Press, 1977.

LEVY, M. N., IANo, T., \& Zieske, H. Effects of repetitive bursts of vagal activity on heart rate. Circulation Research, 1972, 30, 186-195.

Levy, M. N., Martin, P. J., Iano, T., \& Zieske, H. Paradoxical effect of vagus nerve stimulation on heart rate in dogs. Circulation Research, 1969, 25, 303-314.

Levy, M. N., Martin, P. J., Iano, T., \& Zieske, H. Effects of single vagal stimuli on heart rate and atrioventricular conduction. American Journal of Physiology, 1970, 218, 1256-1262.

LEVY, M. N., \& ZIESKE, H. Synchronization of the cardiac pacemaker with repetitive stimulation of the carotid sinus nerve in the dog. Circulation Research, 1972, 30, 634-664.

MonNier, M. Functions of the nervous system (Vol. I). (Chap. 8. Neo-cortical organization of visceral functions). AmsterdamLondon-New York: Elsevier, 1968.

REID, J. V. O. The cardiac pacemaker: Effects of regularly spaced nervous input. American Heart Journal, 1969, 78, 58-64.

SIEGEL, S. Nonparametric statistics for the behavioral sciences. New York: McGraw-Hill, 1956.

WELFORD, N. T. The SETAR: An electronic digital event recorder and its use in a psychophysiological laboratory. In $\mathrm{H}$. Zimmer (Ed.), Computers in psychophysiology. Springfield, ILL: Thomas, 1966.

Wilcoxon, F., \& Wilcox, R. A. Some rapid approximate statistical procedures. New York: Lederle Laboratories, 1964.

\section{NOTES}

1. A related study was described by us in a preliminary report (J. I. Lacey \& B. C. Lacey, 1973), and a comprehensive report is now being prepared for publication. Stimuli were presented only at two points within the cardiac cycle: either simultaneously with the R-wave or $350 \mathrm{msec}$ after the R-wave. Heart period was slower for 350-msec delays. Jennings and Wood (1977) have confirmed this specific finding.

2. It should be noted that Eckberg (1976) plotted the time of stimulation (neck suction) with respect to the subsequent $\mathrm{P}$-wave rather than the $\mathrm{P}$-wave which initiated the stimulus cycle. It is 
not unlikely that the difference in reference points underlies an apparent, rather than a real, discrepancy between Eckberg's findings and ours. For example, a subject with a base HP of $1,000 \mathrm{msec}$ who showed peak inhibition to events occurring $400 \mathrm{msec}$ after the initiating R-wave and a subject with a base HP of $700 \mathrm{msec}$ who showed peak inhibition to events occurring $100 \mathrm{msec}$ after the initiating $\mathrm{R}$-wave would both show identical timing of peak response $(-600 \mathrm{msec})$ with respect to the subsequent R-wave. It may also be of some consequence that the responses Eckberg obtained, with prolonged and large negative pressures, were massive ones.

(Received for publication April 26, 1977; accepted July $21,1977$. 